\title{
Implante de lentes intra-oculares de câmara posterior em olhos fácicos para correção de hipermetropia
}

\author{
Implantation of posterior chamber intraocularlens in phakic eyes for the correction of \\ hyperopia
}

Cristina Moreira Salera ${ }^{1}$ Edgar Emiliano Duarte Servian ${ }^{1}$ Márcia Reis Guimarães ${ }^{2}$ Raul Damásio Castro ${ }^{3}$ Ricardo Queiroz Guimarães ${ }^{4}$

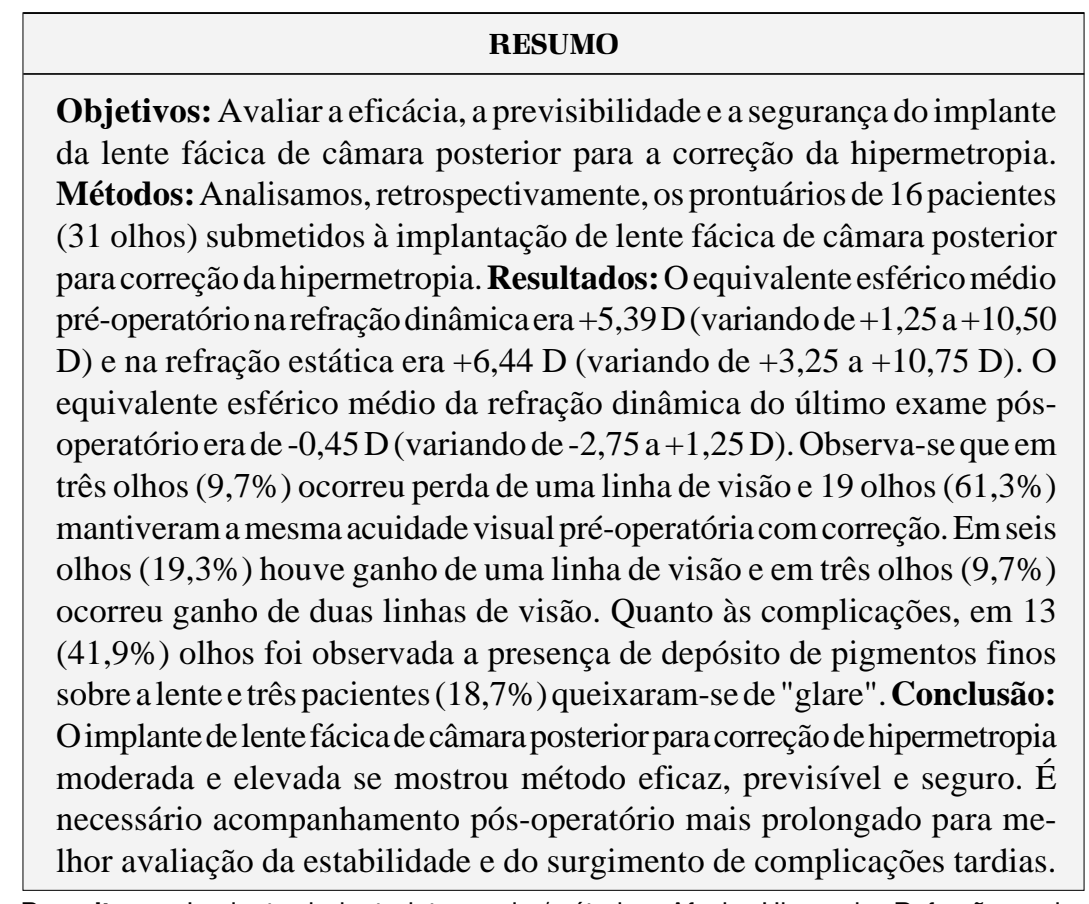

Descritores: Implante de lente intra-ocular/métodos; Afacia; Hiperopia; Refração ocular

\section{INTRODUÇÃO}

As soluções em cirurgia refrativa para correção da hipermetropia envolvem duas principais estruturas anatômicas: a córnea e o cristalino ${ }^{(1)}$. Em função das limitações da correção corneana pelo “excimer laser” no tratamento de altas ametropias, observamos nos últimos anos, um crescente interesse no implante de lentes intra-oculares em olhos fácicos ${ }^{(1-4)}$.

A lente de contato implantável (ICL) ${ }^{\mathrm{TM}}$ (Staar Surgical AG) é uma lente intra-ocular fácica de câmara posterior que deve ser posicionada entre a superfície posterior da íris e a superfície anterior do cristalino ${ }^{(3)}$ (figura 1). Esta lente é fabricada com um polímero de colágeno (Collamer) que apresenta muitas características favoráveis como: alta permeabilidade ao oxigênio e nutrientes, biocompatibilidade, flexibilidade e alto índice de refração $(1,45)^{(4-6)}$.

As vantagens deste tipo de procedimento incluem a sua reversibilidade e a preservação da acomodação ${ }^{(1,3,7-8)}$. Como complicações potenciais deste procedimento podemos esperar encontrar: glaucoma por bloqueio pupilar, glaucoma maligno, glaucoma secundário à neovascularização do ângulo, 
depósito de pigmentos sobre a superfície da lente, "glare", halos, hipocorreção e catarata ${ }^{(1,5,8)}$. Em 1993, pela primeira vez, esta lente de "Collamer" foi implantada em olhos humanos na Europa para a correção de alta miopia ${ }^{(8-9)}$. Entretanto, é importante ressaltar que os primeiros ensaios clínicos das lentes intra-oculares em olhos fácicos de outros materiais para correção de erros refrativos são datados da década de $50^{(4)}$.

Este trabalho tem o objetivo de avaliar a eficácia, a previsibilidade e a segurança do implante de lente fácica de câmara posterior em pacientes com hipermetropia moderada e elevada.

\section{MÉTODOS}

Foram avaliados, retrospectivamente, os dados de 31 olhos (16 pacientes) submetidos ao implante de ICL no período de dezembro de 1996 a agosto de 2000, pelo mesmo cirurgião (Dr. Ricardo Guimarães), para correção refrativa em pacientes com hipermetropia. Os dados analisados foram os seguintes:

- sexo;

- idade;

- acuidade visual sem correção pré-operatória;

- acuidade visual com correção pré-operatória;

- refração dinâmica pré-operatória;

- refração estática pré-operatória;

- acuidade visual sem correção pós-operatória (um dia, 30dias e 90 dias);

- refração dinâmica pós-operatória (um dia, 30 dias e 90 dias);

- complicações;

- outros procedimentos.

Este trabalho faz parte de um protocolo institucional de pesquisa do Hospital de Olhos de Minas Gerais, de caráter experimental e aprovado por uma comissão de ética médica interna.

Os pacientes foram informados sobre o caráter experimental do procedimento, riscos e benefícios, e concordaram em assinar um termo de consentimento.

A avaliação pré-operatória, de acordo com o que foi instituído pelo fabricante no protocolo, incluiu os seguintes exames:

- refração dinâmica e estática;

- medida da pressão intra-ocular;

- medida da profundidade da câmara anterior (ecógrafo A com sonda de contato);

- medida da espessura da córnea (paquímetro ultra-sônico);

- medida do diâmetro corneano (compasso);

- topografia corneana computadorizada de elevação Orbscan (sistema de topografia corneana baseada na luz de fenda e disco de Plácido que mede as elevações e as curvaturas das superfícies anterior e posterior da córnea);

- biomicroscopia;

- fundoscopia;

- microscopia especular (microscópio especular computadorizado sem contato).

O objetivo refracional da cirurgia era a emetropia, levandose em conta que esta lente corrige somente o componente esférico da ametropia.
As especificações da ICL para cada paciente foram determinadas em protocolo definido pelo fabricante, levando-se em conta os dados obtidos no exame pré-operatório.

A ICL é uma lente dobrável, em plataforma e com concavidade posterior para ser implantada na câmara posterior, entre a íris e o cristalino, com injetor próprio.

O diâmetro da lente para hipermetropia varia de 11,00 a $12,00 \mathrm{~mm}$, com zona óptica de 4,8 a 5,8 mm e espessura central de aproximadamente 60 micra, dependendo do poder dióptrico da lente que pode variar de $+3,00$ a $+17,00$ dioptrias. Neste estudo foram utilizados dois modelos de lente: V2 e V3. O modelo V2 da ICL apresentava uma concavidade posterior muito acentuada o que provavelmente dificultava o aporte de nutrientes e a remoção de catabólitos do epitélio lenticular, assim sendo, foi substituída pelo modelo V3.

Com o objetivo de evitar o bloqueio pupilar pós-operatório, foram realizadas duas iridotomias com uma distância de $90^{\circ}$ uma da outra através dos lasers de argônio e YAG, pelo menos uma semana antes da cirurgia, o que previne o depósito de pigmentos sobre a ICL.

Em apenas dois olhos (um paciente) foi realizada a anestesia tópica, nos demais, a anestesia utilizada foi o bloqueio peribulbar. A rotina para a preparação do paciente consistiu em sedação com midazolan (Dormonid ${ }^{\circledR}$ ) 3ml intravenoso e cloridrato de fentanil 0,5 mg (Fentanil $\left.{ }^{\circledR}\right) 1 \mathrm{ml}$ intravenoso. Para a dilatação da pupila utilizou-se uma gota dos seguintes colírios: cloridrato de proximetacaína (Anestalcon ${ }^{\circledR}$ ), tropicamida $\left(\right.$ Mydriacyl $^{\circledR}$ ), cloridrato de fenilefrina $\left(\right.$ Fenilefrina $^{\circledR}$ ) e suprofeno (Procofen ${ }^{\circledR}$ ). A anestesia peribulbar de 29 olhos foi realizada utilizando-se $6 \mathrm{ml}$ de cloridrato de lidocaína $2 \%$ com vasoconstritor (1:400.000). Na anestesia tópica utilizou-se cloridrato de bupivacaína 0,75\% sem vasoconstritor, 20 gotas em 15 minutos. A redução da pressão intra-ocular foi obtida através a utilização do balão de Honan por 20 minutos.

Quanto à técnica cirúrgica, inicialmente duas incisões auxiliares eram realizadas afastadas $180^{\circ}$ uma da outra e a $90^{\circ}$ da incisão principal que era realizada no lado temporal ou no

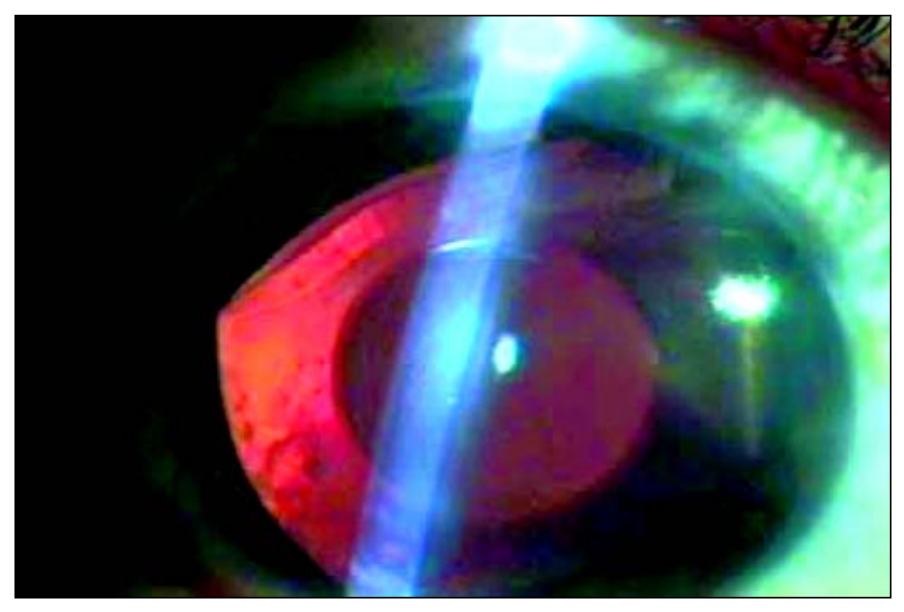

Figura 1 - Exame à lâmpada de fenda mostrando ICL bem posicionada 
meridiano mais curvo com o objetivo de reduzir o astigmatismo, com 3,2 mm de extensão. A seguir, foi injetada substância viscoelástica de alto peso molecular (Healon ${ }^{\circledR}$ ) generosamente dentro da câmara anterior. A ICL era removida do frasco e colocada no injetor fornecido pelo fabricante. Uma vez implantada na câmara anterior, a extremidade distal (footplates) da ICL e, posteriormente a proximal, eram cuidadosamente posicionadas atrás da íris. Uma espátula (ICL Manipulator, Katena Inc.) foi usada para a colocação da lente sob a íris. Um mantenedor de câmara anterior era posicionado em uma das incisões auxiliares e a substância viscoelástica era removida da câmara anterior e do espaço entre a lente e o cristalino com o sistema de irrigação/aspiração. Ao final do procedimento provocavase a miose com carbachol (Miostat ${ }^{\circledR}$ ) 0,01\%, 1 ml diluído 1:1. Foi utilizada sutura para fechamento da incisão apenas naqueles casos cuja incisão era ampliada além de 3,2 mm com o objetivo de se corrigir o astigmatismo. O paciente era examinado na lâmpada de fenda algumas horas após a cirurgia para assegurar que a câmara anterior estivesse com a profundidade adequada, a lente bem posicionada e não houvesse resíduos de viscoelástico. Foi prescrito para uso tópico a associação antibiótico/corticóide (sulfato de neomicina, sulfato de polimixina B e dexametasona) inicialmente na freqüência de quatro vezes ao dia com redução de uma gota a cada semana. O exame completo pós-operatório era realizado no dia seguinte e com 1 , 3, 6, 12, 24 e 36 meses após a cirurgia.

\section{RESULTADOS}

O método de análise estatística utilizado foi o T de Student para amostras pareadas. O grupo estudado era composto por 31 olhos de 16 pacientes, sendo que seis $(37,5 \%)$ eram do sexo feminino e dez (62,5\%) do sexo masculino. Destes pacientes, 15 tiveram a ICL implantada em ambos os olhos e um somente no olho direito. A idade variou de 25 a 44 anos com média igual a 34,2 anos.

O gráfico 1 mostra a distribuição da acuidade visual com e sem correção antes da cirurgia. Pode-se observar que cerca de $68 \%$ dos olhos avaliados possuíam acuidade visual sem correção de 20/100 ou menos. Com o uso da correção mais de $45 \%$ atingiram visão $20 / 20$ e outros $41,9 \%$ ficaram no intervalo entre $20 / 25$ e 20/40, o que totaliza $87,1 \%$ dos casos.

O equivalente esférico médio na refração dinâmica pré-operatória foi de +5,39 dioptrias variando de +1,25 a +10,50 dioptrias (desvio padrão 2,27). Na refração estática pré-operatória o equivalente esférico médio foi de $+5,44$ dioptrias variando de $+3,25$ a +10,75 dioptrias (desvio padrão 1,89). No último exame pós-operatório o equivalente esférico médio na refração dinâmica foi de -0,44 dioptrias variando de $-2,75$ a $+1,25$ dioptrias (desvio padrão 0,89). A ametropia cilíndrica média pré-operatória era $-1,14$ dioptrias variando de 0 a $-3,50$ dioptrias (desvio padrão 0,97) e no pós-operatório, a média foi de-1,28 dioptrias, variando de 0 a -4,00 dioptrias (desvio padrão 1,03).

O gráfico 2 mostra a distribuição da acuidade visual com e sem correção após a cirurgia. Após a cirurgia, mais de $60 \%$ dos casos apresentaram visão 20/40 ou melhor sem correção. Com a correção, no pós-operatório, a proporção de olhos nesta faixa manteve-se $87,1 \%$, como no pré-operatório, porém com $54,8 \%$ com visão $20 / 20$.

No gráfico 3 observa-se a comparação entre os grupos de acuidade visual sem correção antes e após a cirurgia. Registrou-se cerca de $68 \%$ dos casos com visão $20 / 100$ ou pior no pré-operatório e mais de $61 \%$ com visão 20/40 ou melhor no pós-operatório.

Quanto à acuidade visual com correção antes e após a cirurgia, também encontramos uma distribuição semelhante como podemos observar no gráfico 4 .

A tabela 1 mostra a comparação entre as acuidades visuais com correção pré-operatórias e sem correção pós-operatórias $(\mathrm{p}=0,011)$.

A análise da acuidade visual através da perda e do ganho de linhas de visão é um bom parâmetro para verificação da segurança do procedimento. Quando comparamos a acuidade

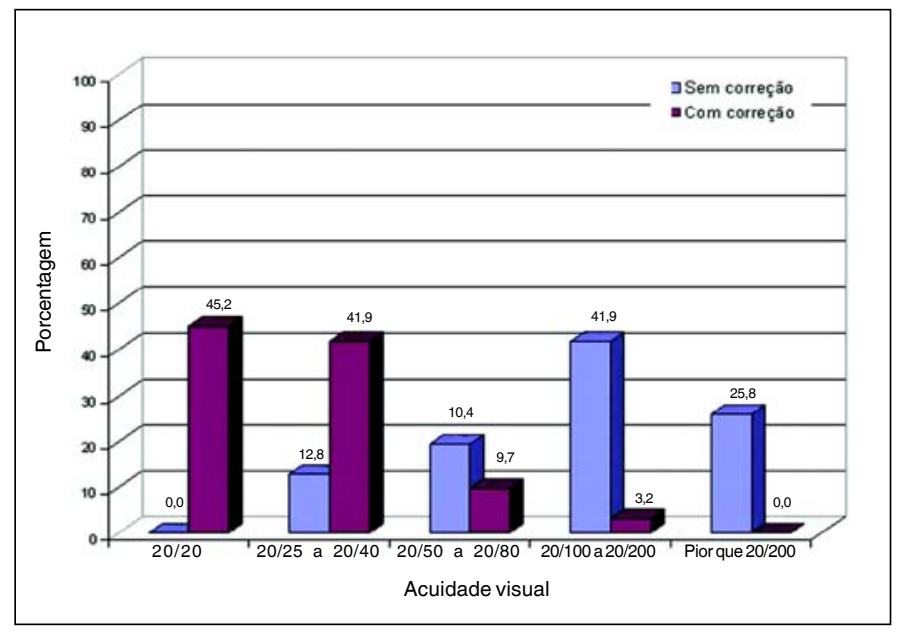

Gráfico 1 - Distribuição da acuidade visual antes da cirurgia

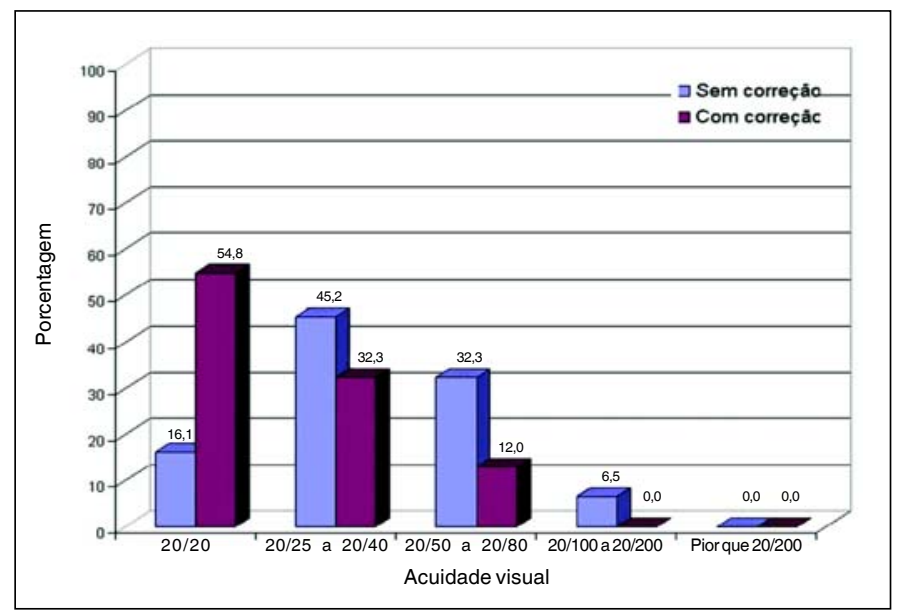

Gráfico 2 - Distribuição da acuidade visual após a cirurgia (90 DPO) 


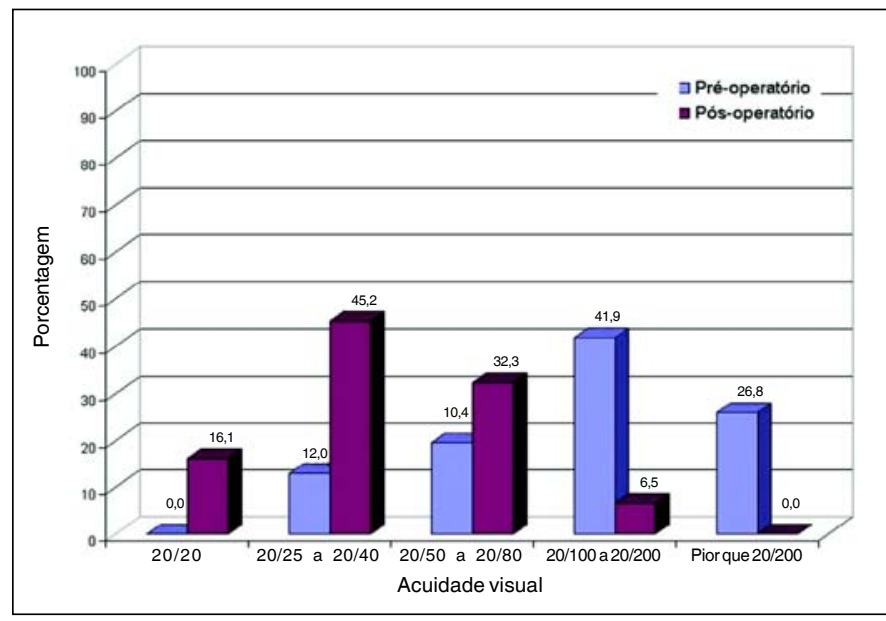

Gráfico 3 - Distribuição da acuidade visual sem correção pré e pósoperatórias

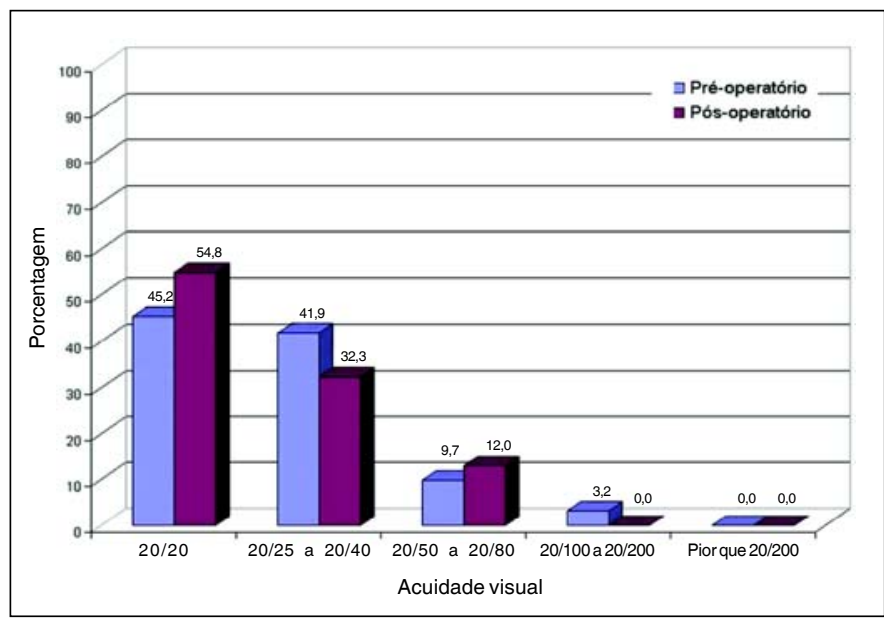

Gráfico 4 - Distribuição da acuidade visual com correção pré e pósoperatórias

\begin{tabular}{|c|c|c|c|c|}
\hline $\begin{array}{l}\text { Acuidade } \\
\text { visual }\end{array}$ & Mínimo & Máximo & Média & DP \\
\hline AV CC pré & $20 / 20$ & $20 / 150$ & $20 / 32$ & $20 / 25$ \\
\hline AV SC pós & $20 / 20$ & $20 / 100$ & $20 / 44$ & $20 / 25$ \\
\hline
\end{tabular}

visual sem correção pré e pós-operatórias, observa-se que não ocorreu perda de linhas de visão em nenhum dos casos e 20 (64,5\%) olhos ganharam mais de três linhas de visão. Já, quando comparamos a acuidade visual com correção pré e pós-operatórias nota-se que três $(9,7 \%)$ olhos perderam uma linha de visão, 19 (61,3\%) olhos mantiveram a mesma acuidade visual, seis $(19,3 \%)$ olhos ganharam uma linha de visão e em três $(9,7 \%)$ olhos houve ganho de duas linhas de visão (gráfico 5). A tabela 2 mostra os dados clínicos dos pacientes.

\section{COMPLICAÇÕES}

Em nosso estudo, a complicação mais comum foi a presença de um leve depósito de pigmentos finos sobre a superfície anterior da lente, encontrado em 13 olhos (41,9\%). Este achado não estava associado a qualquer queixa subjetiva de piora da visão e não houve alteração da pressão intra-ocular e constatação de depósitos no ângulo da câmara anterior.

A segunda complicação mais comum foi a queixa de "glare" no pós-operatório que foi relatada por três pacientes $(18,7 \%)$ sem que houvesse qualquer alteração biomicroscópica que justificasse tal queixa.

Em um (3,2\%) olho, durante o exame realizado no pósoperatório imediato, foi observado que a lente estava parcialmente capturada pela íris sendo indicado o reposicionamento da mesma e evoluindo com leve depósito de pigmentos finos sobre a superfície anterior da lente sem perda de linhas de visão.

Também em um (3,2\%) olho foi detectada a presença de Seidel espontâneo no pós-operatório imediato que persistiu por 24 horas sendo, então, indicada a realização de sutura.

Mesmo com uma média de acompanhamento destes pacientes de 12,1 meses (variando de um mês a 41 meses), não constatamos a formação de opacificação do cristalino.

\section{DISCUSSÃO}

Os olhos hipermétropes, em geral, possuem segmentos anteriores relativamente pequenos, tornando assim, a cirurgia para implante de lente fácica de câmara posterior, um procedimento mais difícil ${ }^{(1,8,10)}$.

Recentes estudos demonstraram um aumento na segurança do procedimento após a realização de duas iridotomias préoperatórias $^{(8,10)}$ colocando, portanto, a cirurgia de implante da lente fácica de câmara posterior como mais uma opção para o arsenal dos cirurgiões refrativos para o tratamento da hipermetropia. Esta técnica cirúrgica apresenta algumas vantagens

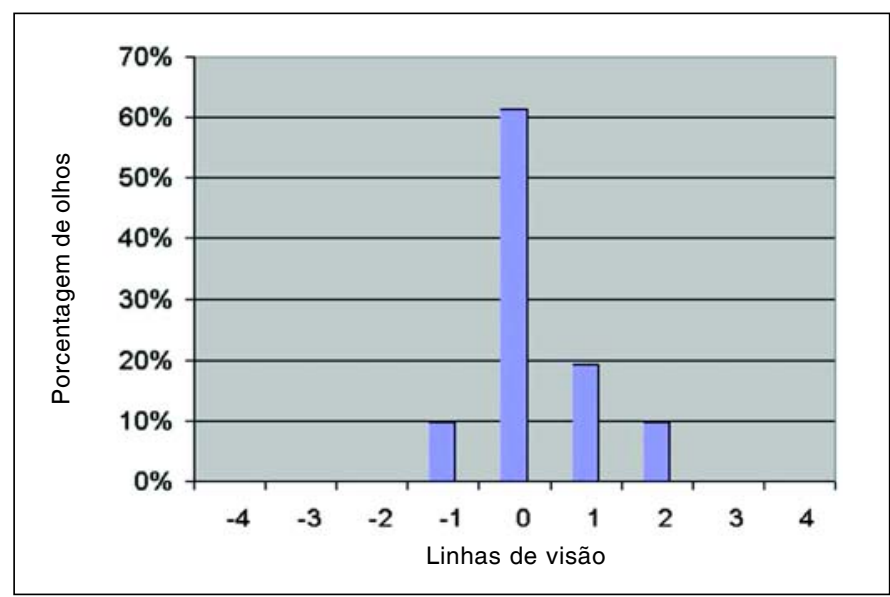

Gráfico 5 - Linhas ganhas ou perdidas (AVCC pré x AVCC pós) 


\begin{tabular}{|c|c|c|c|c|c|c|c|c|}
\hline $\mathbf{N}$ & Sexo & Idade & $\begin{array}{l}\text { AV C/C } \\
\text { Pré-op }\end{array}$ & $\begin{array}{l}\text { Refração } \\
\text { dinâmica } \\
\text { Pré-op }\end{array}$ & $\begin{array}{l}\text { Refração } \\
\text { estática } \\
\text { Pré-op }\end{array}$ & $\begin{array}{l}\text { AV S/C } \\
\text { Pós-op } \\
90 \circ \text { DPO }\end{array}$ & $\begin{array}{l}\text { AV C/C } \\
\text { Pós-op } \\
90^{\circ} \text { DPO }\end{array}$ & $\begin{array}{c}\text { Refração } \\
\text { dinâmica } \\
\text { Pós-op }\end{array}$ \\
\hline 1 & $\mathrm{M}$ & 30 & $20 / 20$ & $+5,75-1,25 \times 180$ & $+8,00-1,25 \times 180$ & $20 / 25$ & $20 / 20$ & $+1,25-1,50 \times 175$ \\
\hline 2 & $\mathrm{M}$ & 30 & $20 / 20$ & $+6,25-1,25 \times 15$ & $+8,25-1,25 \times 15$ & $20 / 30$ & $20 / 25$ & $+0,50-1,50 \times 10$ \\
\hline 3 & $\mathrm{M}$ & 27 & $20 / 20$ & $+5,50-2,00 \times 105$ & $+7,00-2,00 \times 105$ & $20 / 25$ & $20 / 20$ & $+0,50-1,00 \times 120$ \\
\hline 4 & M & 27 & $20 / 20$ & $+5,00-1,00 \times 65$ & $+6,25-1,00 \times 65$ & $20 / 25$ & $20 / 20$ & $+0,75-1,00 \times 55$ \\
\hline 5 & $\mathrm{~F}$ & 43 & $20 / 20$ & $+2,25$ & $+3,25$ & $20 / 20$ & $20 / 20$ & $-0,25$ \\
\hline 6 & $\mathrm{~F}$ & 43 & $20 / 20$ & $+2,25$ & $+3,25$ & $20 / 25$ & $20 / 20$ & PL -0,75x90 \\
\hline 7 & $\mathrm{~F}$ & 38 & $20 / 20$ & $+4,25-1,50 \times 170$ & $+6,50-1,25 \times 170$ & $20 / 40$ & $20 / 20$ & PL -1,75x175 \\
\hline 8 & $\mathrm{~F}$ & 38 & $20 / 40$ & $+5,00-2,25 \times 40$ & $+7,25-2,75 \times 40$ & $20 / 80$ & $20 / 25$ & $+0,25-1,75 \times 40$ \\
\hline 9 & $M$ & 39 & $20 / 25$ & $+3,00-0,25 \times 10$ & $+4,50-0,25 \times 10$ & $20 / 20$ & $20 / 20$ & Plano \\
\hline 10 & M & 39 & $20 / 25$ & $+1,50-0,50 \times 10$ & $+3,75-0,50 \times 10$ & $20 / 20$ & $20 / 20$ & PL $-0,50 \times 180$ \\
\hline 11 & M & 33 & $20 / 30$ & $+6,00-0,50 \times 160$ & $+7,00-0,50 \times 160$ & $20 / 25$ & $20 / 20$ & PL $-0,25 \times 155$ \\
\hline 12 & $M$ & 33 & $20 / 25$ & $+6,00-1,00 \times 180$ & $+7,00-1,00 \times 180$ & $20 / 25$ & $20 / 20$ & $+0,25-0,50 \times 160$ \\
\hline 13 & M & 39 & $20 / 30$ & $+6,25-1,75 \times 170$ & $+7,00-1,50 \times 170$ & $20 / 80$ & $20 / 30$ & $+1,00-2,50 \times 180$ \\
\hline 14 & $M$ & 39 & $20 / 20$ & $+2,75-0,50 \times 180$ & $+3,50-0,50 \times 180$ & $20 / 30$ & $20 / 20$ & $+0,50-1,00 \times 180$ \\
\hline 15 & $\mathrm{~F}$ & 41 & $20 / 30$ & $+9,00-0,75 \times 120$ & $+9,50-0,75 \times 120$ & $20 / 40$ & $20 / 30$ & $-1,25-1,00 \times 160$ \\
\hline 16 & $\mathrm{~F}$ & 41 & $20 / 40$ & $+9,50-0,25 \times 180$ & $+9,50$ & $20 / 60$ & $20 / 40$ & $-2,25-1,00 \times 180$ \\
\hline 17 & M & 38 & $20 / 60$ & $+10,25$ & $+10,75$ & $20 / 100$ & $20 / 80$ & $+1,50-0,50 \times 75$ \\
\hline 18 & M & 38 & $20 / 50$ & $+10,50$ & $+10,50$ & $20 / 60$ & $20 / 50$ & $+1,00$ \\
\hline 19 & $\mathrm{~F}$ & 32 & $20 / 20$ & $+7,00$ & $+7,50$ & $20 / 30$ & $20 / 25$ & $+1,25-0,75 \times 130$ \\
\hline 20 & $\mathrm{~F}$ & 32 & $20 / 150$ & $+7,00-0,75 \times 180$ & $+7,50-0,50 \times 180$ & $20 / 150$ & $20 / 80$ & Plano \\
\hline 21 & $\mathrm{~F}$ & 31 & $20 / 25$ & $+4,50-1,25 \times 10$ & $+6,50$ & $20 / 50$ & $20 / 20$ & PL $-2,00 \times 25$ \\
\hline 22 & $\mathrm{~F}$ & 31 & $20 / 20$ & $+4,00-1,00 \times 180$ & $+5,00-1,00 \times 180$ & $20 / 25$ & $20 / 20$ & PL $-1,25 \times 180$ \\
\hline 23 & M & 32 & $20 / 20$ & $+5,75-1,50 \times 165$ & $+6,25-1,00 \times 165$ & $20 / 30$ & $20 / 20$ & $-0,75-1,75 \times 165$ \\
\hline 24 & M & 32 & $20 / 20$ & $+6,25-1,50 \times 15$ & $+6,50-1,25 \times 15$ & $20 / 20$ & $20 / 20$ & PL -1,50x15 \\
\hline 25 & $\mathrm{~F}$ & 25 & $20 / 25$ & $+4,50-0,50 \times 180$ & $+6,50-0,50 \times 180$ & $20 / 100$ & $20 / 25$ & $-1,75-1,25 \times 165$ \\
\hline 26 & M & 44 & $20 / 70$ & $+8,75-3,00 \times 100$ & $+8,75-3,00 \times 100$ & $20 / 60$ & $20 / 50$ & $+1,00-3,25 \times 95$ \\
\hline 27 & M & 44 & $20 / 30$ & $+8,50-2,50 \times 89$ & $+8,50-2,50 \times 89$ & $20 / 60$ & $20 / 30$ & $+0,50-2,25 \times 75$ \\
\hline 28 & M & 36 & $20 / 20$ & $+7,50-3,25 \times 180$ & $+8,25-3,25 \times 180$ & $20 / 70$ & $20 / 20$ & $+0,75-3,75 \times 180$ \\
\hline 29 & M & 36 & $20 / 20$ & $+7,50-3,50 \times 180$ & $+8,25-3,50 \times 180$ & $20 / 70$ & $20 / 20$ & $+0,75-4,00 \times 175$ \\
\hline 30 & $M$ & 31 & $20 / 30$ & $+6,75-1,00 \times 180$ & $+7,25-1,00 \times 180$ & $20 / 30$ & $20 / 25$ & $+0,50-0,75 \times 170$ \\
\hline 31 & $M$ & 31 & $20 / 25$ & $+5,75-0,75 \times 180$ & $+6,50-0,75 \times 180$ & $20 / 20$ & $20 / 25$ & PL $-0,75 \times 175$ \\
\hline
\end{tabular}

quando comparada a outros procedimentos como, por exemplo, sua reversibilidade (a lente pode ser removida ou substituída) ${ }^{(1-4)}$, sua estabilidade (não depende do processo cicatricial para o resultado refrativo final $)^{(3-4)}$, boa qualidade de visão (não ocorre alteração na estrutura e no relevo corneano) $)^{(1,4)}$ requer um investimento de custo reduzido (apesar do elevado custo da lente, não requer nenhum equipamento sofisticado) ${ }^{(3-4)}$, além de preservar a acomodação do paciente ${ }^{(1-3,8)}$.

Em contrapartida, por se tratar de um procedimento intraocular, tem maior risco para complicações como catarata, glaucoma e endoftalmite (1,4,7,10). $^{\text {. }}$

A lente fácica de câmara posterior corrigiu a hipermetropia em nosso grupo estudado. A análise mostrou uma melhora significativa no equivalente esférico dinâmico $(\mathrm{p}<0,01)$. Apesar de alguns olhos terem aceitado no pós-operatório lentes negativas, a média que era $+5,39 \pm 2,25$ dioptrias, ficou próxima

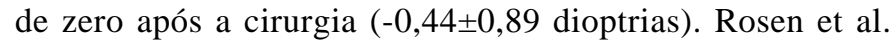

obtiveram resultado parecido em seu grupo de hipermétropes, oito dos seus nove pacientes ficaram entre $-0,50$ e $+0,50$ de equivalente esférico ${ }^{(1)}$. Davidorf et al. também encontraram resultados semelhantes e ainda relataram que uma das limitações que interferem na previsibilidade do implante de lente fácica de câmara posterior para hipermetropia é o cálculo do valor dióptrico da lente cujas fórmulas ainda devem ser aperfeiçoadas $^{(8)}$. Este mesmo autor encontrou uma boa estabilidade da refração em 12 meses de acompanhamento pós-operatório ${ }^{(8)}$.

A eficácia do procedimento pode ser verificada pela observação de que $61,3 \%$ dos olhos apresentaram acuidade visual sem correção pós-operatória de 20/40 ou melhor enquanto que, antes da cirurgia, 87,1\% apresentavam esta mesma acuidade visual, porém com correção. De fato, houve uma diferença estatisticamente significativa na acuidade visual sem correção $(\mathrm{p}<0,01)$ antes e após a cirurgia. Quando comparamos as médias entre a acuidade visual com correção pré-operatória e a 
acuidade visual sem correção pós-operatória, encontramos uma diferença significativa, sendo que a visão corrigida antes da cirurgia era melhor, em média, que a acuidade visual sem correção após a cirurgia $(\mathrm{p}=0,011)$. Resultado semelhante também foi encontrado ${ }^{(8)}$.

Quando analisamos a proporção de linhas ganhas ou perdidas, pode-se observar que o ganho de linhas da acuidade visual não corrigida foi muito significativo, sendo que em quase $65 \%$ dos olhos observou-se um ganho de mais de três linhas de visão e somente 3,2\% mantiveram a mesma acuidade visual. Para a visão corrigida, a maioria (61,3\%) manteve-se da mesma forma e houve perda de uma linha em aproximadamente $10 \%$ dos olhos sem nenhuma causa específica para esta perda. Outros autores $^{(1,8)}$ também encontraram um olho em que ocorreu perda de uma ou mais linhas de visão na acuidade visual pós-operatória com correção, sendo que, neste último a perda de linhas deveu-se à ocorrência de um glaucoma de ângulo fechado secundário.

Quanto à tentativa de redução do astigmatismo através da confecção da incisão principal no meridiano mais curvo, não foi observada uma diferença estatisticamente significativa comparando-se os valores antes e após a cirurgia $(\mathrm{p}=0,09)$.

No que diz respeito às complicações, a mais comum, de acordo com a literatura, era o bloqueio pupilar pós-operatório, que teve a sua incidência reduzida após a realização das iridotomias profiláticas ${ }^{(2,4,8,10)}$. Em nosso estudo não ocorreram casos de bloqueio pupilar pós-operatório. Davidorf et al encontraram uma incidência de 12,5\% deste tipo de complicação em olhos hipermétropes ${ }^{(8)}$. Este mesmo autor, em seu estudo sobre lentes fácicas em olhos míopes, encontrou uma incidência de 4,8\%. Esta diferença se deve, provavelmente, ao menor comprimento axial dos olhos hipermétropes ${ }^{(8)}$. A propósito, olhos com câmara anterior menor que 2,8 mm apresentam uma contra-indicação absoluta ao implante de lente fácica ${ }^{(10)}$.

Nossa complicação mais comum foi o depósito de pigmentos finos sobre a lente, encontrado em 41,9\% dos olhos (13 olhos). Este depósito de pigmento está provavelmente relacionado com o procedimento cirúrgico uma vez que este depósito não é progressivo $^{(8)}$. Apesar da presença deste depósito, nenhum caso de glaucoma foi detectado, assim como em outros relatos da literatu$\mathrm{ra}^{(1,7-8,10)}$. Entretanto, poderíamos encontrar casos de glaucoma pigmentar com o aumento do segmento pós-operatório. Um dos métodos para evitar estes depósitos seria adotar um prazo maior entre a realização das iridotomias e a cirurgia além da implementação da técnica não rotacional do implante da lente ${ }^{(8)}$.

A nossa segunda complicação mais comum, a queixa de "glare", encontrada em seis olhos (19,3\%) também foi relatada em outros estudos ${ }^{(1-2,8)}$ sendo que um destes associou esta queixa com a presença de descentração da lente ${ }^{(8)}$.

Em nosso estudo não encontramos casos de catarata após o implante da lente fácica. Fink et al encontraram três casos de catarata em uma série de 63 olhos $^{(2)}$. O principal fator de risco para a formação da catarata parece ser a proximidade entre a lente e o cristalino ${ }^{(2,4-5)}$. Entretanto, outros mecanismos podem ser relacionados como o trauma do cristalino durante a implan- tação da lente, alterações do metabolismo lenticular causados pela presença da lente ou pelos restos de substância viscoelástica ${ }^{(1,4,10)}$. Este tipo de complicação compromete o resultado da cirurgia mas pode ser corrigida através da remoção da ICL e a substituição do cristalino opacificado por uma lente de câmara posterior para afacia ${ }^{(4,10)}$. A medida do diâmetro branco a branco do olho nos indica uma medida indireta da distância sulco a sulco ${ }^{(2)}$, sendo, portanto, um importante exame préoperatório cuja finalidade é auxiliar na determinação do diâmetro da lente que, caso não esteja adequado, a lente pode apoiar sobre o cristalino predispondo ocorrência de opacificação do cristalino $^{(4)}$.

Sanders et al. compararam os resultados do PRK e do implante de ICL para correção da hipermetropia baixa e concluíram que a previsibilidade entre os dois métodos é semelhan$\mathrm{te}^{(10)}$. Quanto maior o grau de hipermetropia a ser corrigido, piores eram a previsibilidade e a acuidade visual sem correção com o PRK. Assim como o PRK, quando se compara o LASIK e a ICL, a previsibilidade entre os dois procedimentos também é semelhante quando se trata de graus menores de hipermetropia. Na hipermetropia moderada a alta o implante de ICL mostrou melhor previsibilidade. Ao comparar a extração do cristalino transparente com o implante de ICL, o primeiro procedimento mostrou relevante segurança e eficácia. As limitações para este procedimento incluíam a dificuldade no cálculo do poder da lente e a perda da acomodação. A preservação da acomodação é a maior vantagem do implante de ICL sobre a extração do cristalino transparente ${ }^{(10)}$.

Portanto, assim como nas publicações mundiais, nossos resultados foram satisfatórios quanto à eficácia, previsibilidade e segurança do procedimento para correção da hipermetropia. Entretanto, apesar dos bons resultados, um maior seguimento pós-operatório é necessário para melhor avaliação do procedimento quanto à sua estabilidade e as suas complicações tardias.

\section{ABSTRACT}

Purpose: To examine the efficacy, predictability and safety of posterior chamber phakic intra-ocular lens implantation in patients with hyperopia. Methods: The authors analyzed retrospectively 16 patients (31 eyes) submitted to the implantation of a posterior chamber phakic lens for the correction of hyperopia. Results: The mean preoperative spherical equivalent manifest refraction was $+5.39 \mathrm{D}$ (ranging from +1.25 to $+10.50 \mathrm{D}$ ) and cycloplegia refraction was $+6.44 \mathrm{D}$ (ranging from +3.25 to $+10.75 \mathrm{D}$ ). The mean postoperative spherical equivalent at the last visit was -0.44D (ranging from -2.75 to +1.25D). On comparing the preoperative and postoperative corrected visual acuity the results showed that three eyes (9.7\%) lost one line of visual acuity, 19 eyes (61.3\%) kept the same preoperative corrected visual acuity. Six eyes (19.3\%) gained one line of visual acuity and three eyes (9.7\%) gained two lines. Regarding complications, in 13 eyes (41.9\%) a mild amount of 
pigment deposition on the phakic IOL anterior surface was observed and three patients (18.7\%) had complaints of glare. Conclusion: Posterior phakic lens is an effective, predictable and safe method for the correction of mild to severe hyperopia. Visual acuity improvement was significant and a longer follow-up will help to better address long-term stability of the results and the incidence of complications.

Keywords: Lens implantation, intraocular/methods; Aphakia; Hyperopia; Refraction, ocular

\section{REFERÊNCIAS}

1. Rosen E, Gore C. Staar Collamer posterior chamber phakic intraocular lens to correct myopia and hyperopia. J Cataract Refract Surg 1998;24:596-606.

2. Fink AM, Gore C, Rosen E. Cataract development after implantation of the
Staar Collamer posterior chamber phakic lens. J Cataract Refract Surg 1999; 25:278-82.

3. Visessook N, Peng Q, Apple DJ, Gerl R, Schmickler S, Schoderbek RJ, Guindi A. Pathological examination of an explanted phakic posterior chamber intraocular lens. J Cataract Refract Surg 1999;25:216-22.

4. Guimarães RQ, Castro R, Navarro MP, Guimarães MR. Lente fácica de câmara posterior para correção da miopia. Arq Bras Oftalmol 2001;64:21-6.

5. Trindade F, Pereira F. Cataract formation after posterior chamber phakic intraocular lens implantation. J Cataract Refract Surg 1998;24:1661-3.

6. Brown DC, Grabow HB, Martin RG, Rowen SL, Shepherd JR, Williamson $\mathrm{CH}$, Ziémba SL. Staar Collamer intraocular lens: clinical results from the phase I FDA core study.

7. Pershin KB, Pashinova NF. Refractive surgery for hyperopia. J Refract Surg 2000;16:S242-6.

8. Davidorf JM, Zaldivar R, Oscherow S. Posterior chamber phakic intraocular lens for hyperopia of +4 to +11 diopters. J Refract Surg 1998;14:306-11.

9. Zaldivar R, Davidorf JM, Oscherow S. Posterior chamber phakic intraocular lens for myopia of -8 to -19 diopters. J Refract Surg 1998;14:294-305.

10. Sanders DR, Martin RG, Brown DC, Shepherd J, Deitz MR, DeLuca M. Posterior chamber phakic intraocular lens for hyperopia. J Refract Surg 1999; 15:309-15.

\section{CONGRESSO DA}

\section{Sociedade BrasileIra de}

\section{Administração em Oftalmologia - SBaO}

\section{9 e 10 de Julho de 2004 São Paulo - SP}

Informações: tel./fax (11) 3266-4538

e-mail: sbao@sbao.com.br

home page: www.oftalmo.org.br 